\title{
Detection of surface damage in ceramics by photothermal and photoacoustic techniques
}

H. Zhang, S. Gissinger, G. Weides and U. Netzelmann

Fraunhofer-Institute for Nondestructive Testing (IzfP), University, Bldg. 37, 66123 Saarbrücken, Germany

\begin{abstract}
Cracks produced by indenters in zirconia-TiC ceramic and surface defects in $\mathrm{SiSiC}$ ceramic generated by machining were imaged by photothermal radiometry, mirage effect, photoacoustic microscopy with gas cell and piezoelectric detection and scanning acoustic microscopy. The different appearance of the artificial cracks is studied in comparison.
\end{abstract}

\section{INTRODUCTION}

For the development of economic production techniques for ceramic components, one is looking for high resolution nondestructive methods allowing to detect and characterise surface damage. We have studied surface damage produced by indenters and by different machining techniques. Extensive work on cracks in ceramics has been performed by photoacoustic $\left({ }^{1-5}\right)$, mirage $\left({ }^{4,6,7}\right)$ and SEAM $\left({ }^{8}\right)$ techniques. In contrast to the previous work, the emphasis was on a direct comparison of photoacoustic and photothermal images obtained by the different methods. The results were also compared with images obtained by scanning acoustic microscopy (SAM) at $1 \mathrm{GHz}$.

\section{SAMPLES}

A first sample was a ceramic consisting of $70 \%$ zirconia and $30 \% \mathrm{TiC}$. It was optically opaque and had a thermal diffusivity of $2.0 \cdot 10^{-6} \mathrm{~m}^{2} / \mathrm{s}$ (measured by photothermal deflection). Vickers indentations with forces of about $100 \mathrm{~N}$ have been produced on the previously polished surfaces. The second sample was an optically opaque silicon infiltrated silicon carbide ( $\mathrm{SiSiC}$ ) ceramic with different surface machining conditions.

\section{MEASUREMENTS AND RESULTS ON ARTIFICIAL CRACKS}

Amplitude and phase images were obtained by photothermal radiometry (PTR), by photothermal deflection (PTD) measurements, by photoacoustic microscopy using piezoelectric detection (PA-P), and by a photoacoustic gas cell with microphone detection (PA-M). All measurements except PA-P were performed at a modulation frequency of $1 \mathrm{kHz}$.

The edge of the pyramid of a Vickers indentation shown in Fig. 1 is $80 \mu \mathrm{m}$ long, the median cracks starting at the edges can be observed over a length of $100 \mu \mathrm{m}$ in the optical microscope. The area imaged in Fig. 1 is $600 \mu \mathrm{m} \times 600 \mu \mathrm{m}$. 
Fig. 1a (left): PTR amplitude image of the indentation $(600 \mu \mathrm{m} \mathrm{x}$ $600 \mu \mathrm{m})$

Fig. 1b (right): PTR phase image
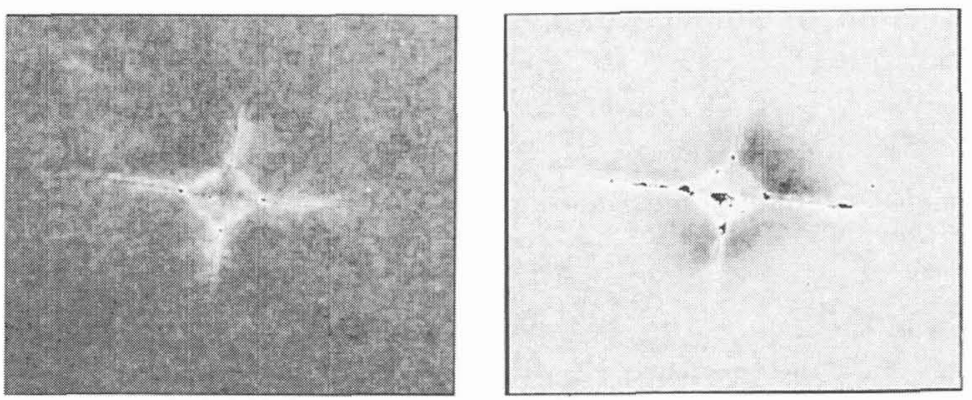

Fig. 1c (left): PA-M amplitude image

Fig. 1d (right): PA-M phase image
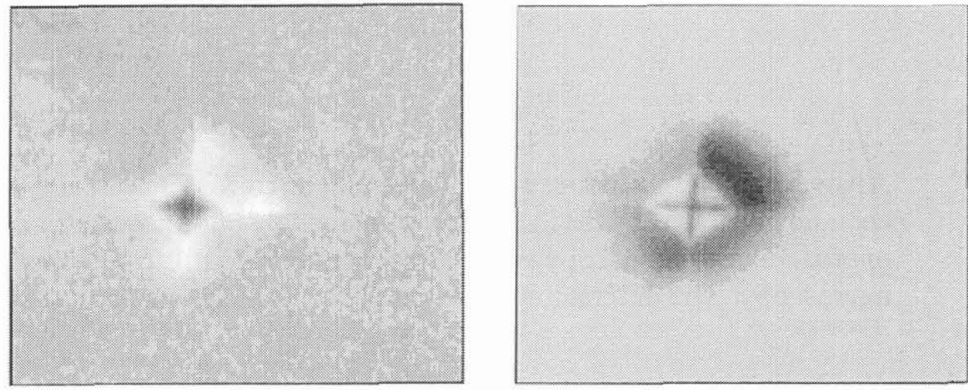

Fig. Ie (left) PA-P amplitude image at $62.2 \mathrm{kHz}$

Fig. 1f (right) PA-P amplitude image at $88.0 \mathrm{kHz}$

Fig. 1g (left): PTD transverse deflection amplitude image

Fig. 1h (right): SAM image at $1 \mathrm{GHz}$
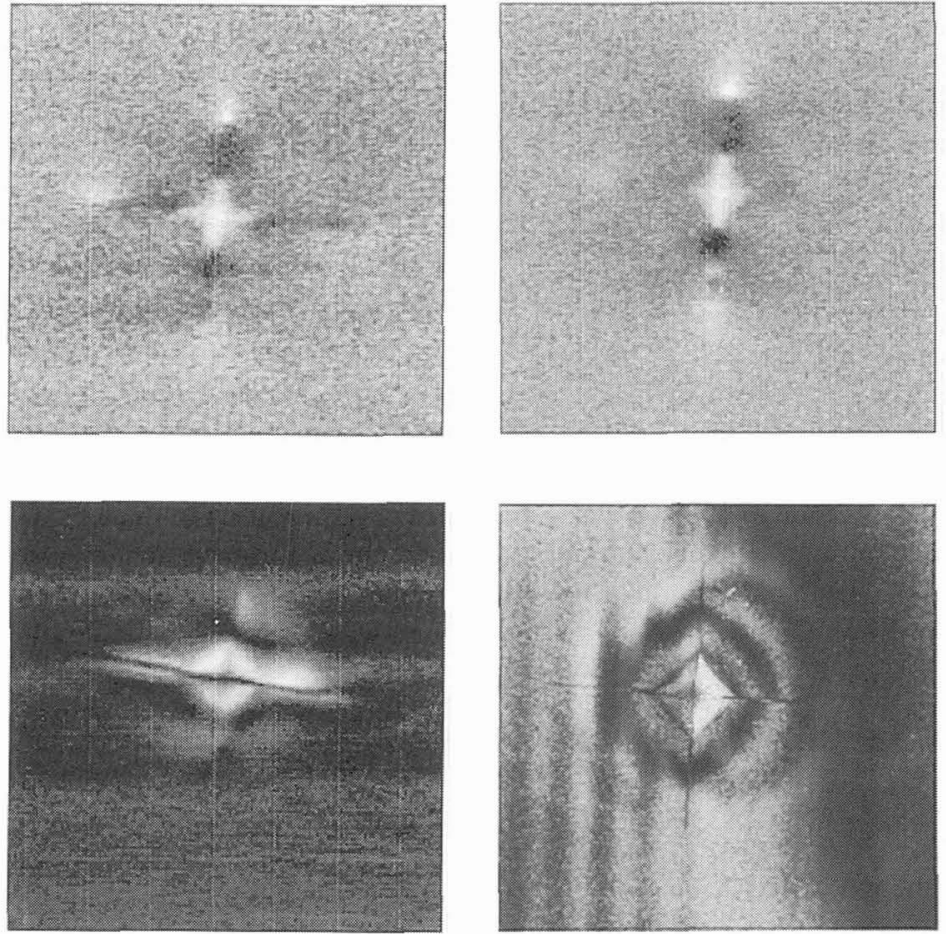


\subsection{Photothermal radiometry (PTR)}

The PTR measurements were obtained by an ALADIN P1 photothermal microscope (Siemens KWU, Offenbach) with a heating beam radius of $3.5 \mu \mathrm{m}$. The median cracks are appearing clearly in the amplitude (Fig. 1a) and phase images (Fig. 1b). In the upper right quadrant, a strong phase minimum of 15 degrees is observed, which is attributed to a lateral crack close to the surface. At lower modulation frequencies, it also appears in the amplitude image.

\subsection{Photoacoustic microscopy by microphone detection (PA-M)}

The PA-M amplitude (Fig. 1c) and phase (Fig. 1d) images were also obtained on the ALADIN microscope, but with the microphone signal of a photoacoustic cell as input for the lock-in amplifier. The phase minimum is observed as well. The lateral resolution obtained, however, is less than in the PTR measurement at the same modulation frequency. One reason for this is that the gas cell acts as an integrating detector for the surface temperature distribution.

\subsection{Photoacoustic microscopy by piezoelectric detection (PA-P)}

Piezoelectric acceleration sensors (Bruel and Kjaer, Denmark) operated at a resonance frequency of the system sample-transducer were employed. The PA-P amplitude images obtained at $62.2 \mathrm{kHz}$ (Fig. le) and at $88 \mathrm{kHz}$ (Fig. 1f) are showing alternating maxima and minima along the cracks, which have been observed in scanning electron acoustic microscopy in a similar way $\left(^{8}\right)$. Depending on the modulation frequency and the excited vibration mode, the horizontal cracks are visible (Fig. 1e) or not visible (Fig. 1f).

\subsection{Photothermal deflection (PTD) measurements}

The PTD images give a contrast comparable to PA-M in the normal deflection signal and, as expected, a very good contrast in the transverse deflection (Fig. 1g). The anisotropy of detection given by the orientation of the probe beam, which passed the sample in the horizontal direction in Fig. 1g, is obvious.

\subsection{Scanning acoustic microscopy (SAM)}

At the experimental parameters used in this work, no thermal technique was able to detect the median cracks in regions where they were not visible by the optical microscope. This is in contrast to the possibilities of the SAM (Fig. 1h), which allows to detect the cracks with an excellent resolution beyond the optically visible length. Water contact for acoustic coupling is necessary. The image was taken at 1 $\mathrm{GHz}$ with a slightly defocused lens, showing the lateral crack by interference fringes.

\section{DETECTION OF SURFACE DAMAGE AND SiC PHASES IN SiSiC CERAMICS}

Images of the bottom of wide grooves machined into $\mathrm{SiSiC}$ have been taken by PTR and PA-P. A typical PTR amplitude image at a frequency of $3000 \mathrm{~Hz}$ is shown in Fig. 2a. The scanning area is $1 \mathrm{~mm} \times 1 \mathrm{~mm}$. Regions of reduced temperature oscillation appearing in dark are clearly observed. These phases are presumed to be $\mathrm{SiC}$ grains inside the Si matrix. Surface damage usually appears as bright spots and can be therefore be separated from the $\mathrm{SiC}$ grains. Images taken at lower frequencies do show the same number of spots, and the lateral resolution of the images remains almost the same, showing the extreme-near-field character of the measurement. The contrast between the $\mathrm{Si}$ and $\mathrm{SiC}$ phases decreases with decreasing frequency, as expected by a theoretical analysis. Fig. $2 b$ shows a PA-P amplitude image at a frequency of $58 \mathrm{kHz}$. The scan area is the same as in Fig. 2a. The dark zone on the left is due to a curvature at the edge of the groove. The $\mathrm{SiC}$ phases appearing in PTR can not be observed. However, below the centre of the image, a crack-like flaw is revealed. The flaw is considered to be located in a depth larger than the thermal wavelength and the non-thermal acoustic nearfield effect is responsible for the contrast. 

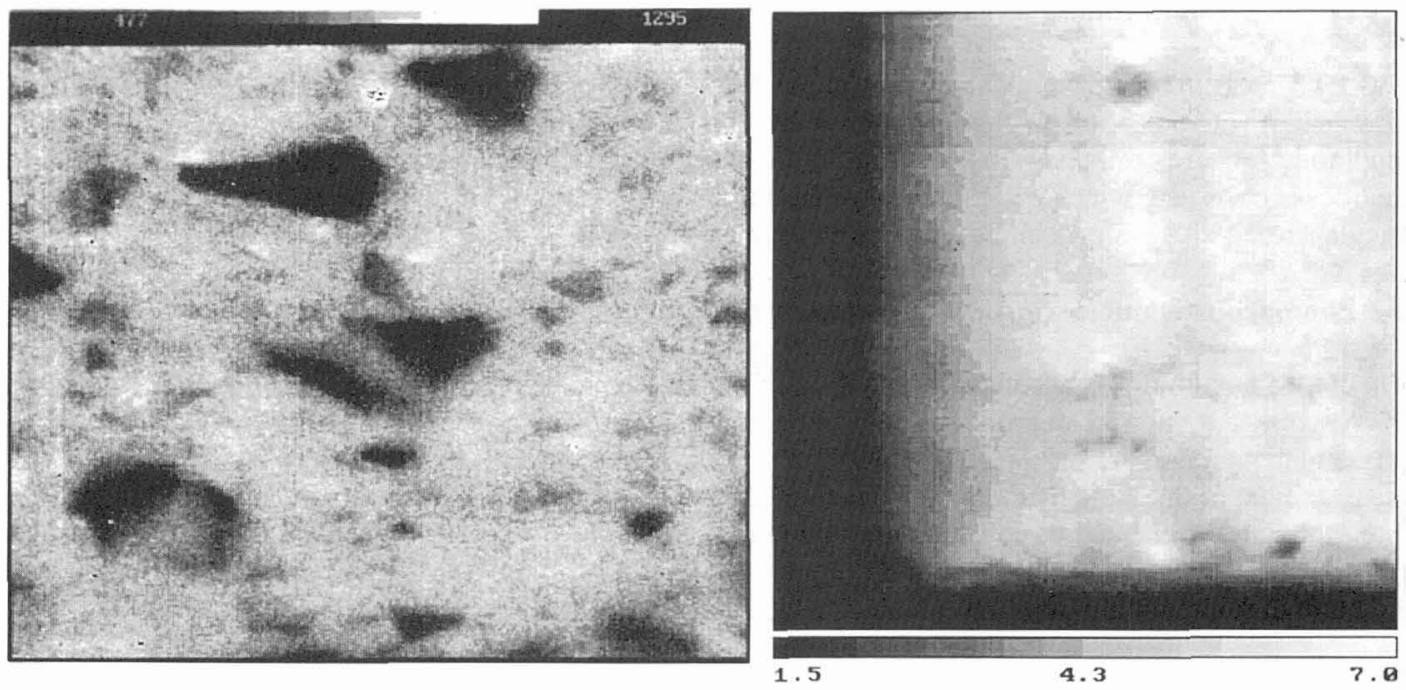

Fig. 2a: PTR amplitude image (1 $\mathrm{mm} \times 1 \mathrm{~mm})$ of the SiSiC ceramic sample
Fig. 2b: PA-P image of the same area as shown in Fig. 2a.

\section{CONCLUSION}

All thermal wave techniques were able to show the artificially produced cracks. However, the cracks are appearing in a characteristic manner for each of the methods. The median cracks were shown with the best lateral resolution by PTR. The best contrast was obtained by transverse PTD, but the method is difficult to apply due to the difficult beam alignment and due to its sensitivity to curvature of the sample surface. Lateral cracks near the surface could be detected by PTR, PTD and PA-M. The lateral resolution of all thermal wave images is usually not comparable to the capability of SAM where the detectability of surface cracks can even compete with the scanning electron microscope $\left({ }^{9}\right)$. The PA-P images reveal mainly the elastic inhomogeneity inside the sample. However, the practical application of this method is often restricted by the difficulty to find proper operation frequencies.

Acknowledgement:

A part of this work was financed by German Minister of Research and Technology under the Sino-German exchange program through the DLR, Cologne:

\section{REFERENCES}

[1] Wong, Y. H., Thomas, R. L., Hawkins, G. F., Appl. Phys. Lett. 32, 538 (1978)

[2] Kandelwal, P., Heitman, P., Silversmith A., Wakefield, T., Appl. Phys. Lett. 37 (1980) 779

[3] Kuo, P. K., Favro, L. D., Inglehardt, L. J., Thomas, R. L., J. Appl. Phys. 53, 1258 (182)

[4] Grice, K. R., Inglehardt, L., Favro, L., Kuo, P. K., and Thomas, R. L., J. Appl. Phys. 54, 6245 (1983)

[5] Hoffmann, B. and Arnold, W., in: Non-Destructive Testing, Boogard, J. and van Dijk, G. M. (eds.), (Elsevier, Amsterdam 1989), p. 1573

[6] Murphy, J. C. and Aamodt, L. C., Appl. Phys. Lett. 39, 519 (1981)

[7] Rantala, J., Hartikainen, J., and Jaarinen, J., Appl. Phys. A 50 (1990) 465

[8] Cantrell, J., Qian, M., Ravichandran, M., Knowles, K., Appl. Phys. Lett. 57 (1990) 1870

[9] Briggs, G. A. D., Jenkins, P. J., and Hoppe, M., J. of Microscopy 159 (1990) 15 